\title{
Identification of a novel Candida metapsilosis isolate suggests ongoing hybridization.
}

Caoimhe E. O’Brien*, Bing Zhai ${ }^{\dagger, \star \S}$, Mihaela Ola*, , Eoin Ó Cinnéide**, Ísla O’Connor*, Thierry Rolling ${ }^{\dagger, \star}$, Edwin Miranda ${ }^{\dagger, \dagger}$, N. Esther Babady ${ }^{\dagger,+}$, Tobias M. Hohl ${ }^{\dagger,+, \dagger^{\dagger}}$ and Geraldine Butler*.

*School of Biomolecular and Biomedical Science, Conway Institute, University College Dublin, Belfield, Dublin 4, Ireland.

${ }^{\dagger}$ Infectious Disease Service, Department of Medicine, Memorial Sloan Kettering Cancer Center, New York, NY, USA

Immunology Program, Sloan Kettering Institute, Memorial Sloan Kettering Cancer Center, New York, NY, USA

${ }^{* *}$ School of Medicine, Conway Institute, University College Dublin, Belfield, Dublin 4, Ireland.

†'Department of Medicine, Weill Cornell Medical College, New York, NY, USA

${ }^{\S}$ Current addresses: BZ: CAS Key Laboratory of Quantitative Engineering Biology, Shenzhen Institute of Synthetic Biology, Shenzhen Institutes of Advanced Technology, Chinese Academy of Sciences, Shenzhen, China. MO: Endocrine Oncology Research Group, Department of Surgery, Royal College of Surgeons in Ireland, Dublin, Ireland.

Data availability: The raw Illumina data for C. metapsilosis MSK606, MSK607, MSK798, MSK801 and MSK414 are available at accession numbers XXXXX (awaiting accession number). The raw MinION data for $C$. metapsilosis MSK414 is available at accession number SRR15054248, and the genome assembly is available at BioProject PRJNA730502 (Accession number JAHFZM000000000). 
Running title: Ongoing hybridization in C. metapsilosis

Keywords: Candida, genomics, hybridization, $\mathrm{LOH}$, mating type-like loci

Corresponding author: Geraldine Butler, School of Biomolecular and Biomedical Science,

Conway Institute, University College Dublin, Belfield, Dublin 4, Ireland. Phone: +353-1-

7166885 (for contact out of office, use +353-86-8315311) Email: gbutler@ucd.ie 


\begin{abstract}
Candida metapsilosis is a member of the C. parapsilosis species complex, a group of opportunistic human pathogens. Of all the members of this complex, C. metapsilosis is the least virulent, and accounts for a small proportion of invasive Candida infections. Previous studies established that all C. metapsilosis isolates are hybrids, originating from a single hybridization event between two lineages, parent A and parent B. Here, we use MinION and Illumina sequencing to characterize a $C$. metapsilosis isolate that originated from a separate hybridization. One of the parents of the new isolate is very closely related to parent A. However, the other parent (parent C) is not the same as parent B. Unlike C. metapsilosis AB isolates, the C. metapsilosis AC isolate has not undergone introgression at the Mating Typelike Locus. In addition, the $\mathrm{A}$ and $\mathrm{C}$ haplotypes are not fully collinear. The C. metapsilosis $\mathrm{AC}$ isolate has undergone Loss of Heterozygosity $(\mathrm{LOH})$ with a preference for haplotype A, indicating that this isolate is in the early stages of genome stabilization.
\end{abstract}




\section{INTRODUCTION}

Candida metapsilosis is a rare opportunistic pathogen of humans (Gomez-Lopez et al. 2008; Lockhart et al. 2008a; Silva et al. 2009). It is a member of the Candida parapsilosis species complex, a small clade of related organisms that includes $C$. metapsilosis, Candida orthopsilosis and C. parapsilosis sensu stricto, all of which cause infection in humans (Tavanti et al. 2005). Within this group, C. parapsilosis is the most common cause of candidiasis, whereas $C$. metapsilosis is the least common, with an incidence ranging from 0.6 - 6.9\% of cases of invasive candidiasis (Gomez-Lopez et al. 2008; Lockhart et al. 2008a; Silva et al. 2009; Cantón et al. 2011; Bonfietti et al. 2012; Bertini et al. 2013). However, isolates from the C. parapsilosis species complex are commonly misidentified, which may have led to an underestimation of the frequency of $C$. orthopsilosis and C. metapsilosis (Tavanti et al. 2007; Lockhart et al. 2008b; Bonfietti et al. 2012). In recent years, it has been suggested that the incidence of $C$. orthopsilosis and C. metapsilosis infection is increasing, although this may be due to increased awareness of the species differentiation (Lockhart et al. 2008a).

Few C. metapsilosis isolates secrete virulence-associated factors such as lipases or aspartic proteinases in comparison to C. parapsilosis (Németh et al. 2013). Whereas C. parapsilosis sensu stricto is commonly associated with infections in neonates, C. metapsilosis is rarely associated with neonatal infection and appears to affect adults predominantly (Cantón et al. 2011). There is no evidence of widespread resistance to any antifungal drugs in $C$. metapsilosis and most isolates tested thus far are susceptible to antifungals (Gomez-Lopez et al. 2008). There is some suggestion that $C$. metapsilosis may be a human commensal, and it has been isolated from the oral cavity of healthy individuals (Ghannoum et al. 2010).

Although all members of the $C$. parapsilosis species complex have diploid genomes, $C$. parapsilosis isolates are highly homozygous with, on average, 0.06 heterozygous SNPs per $\mathrm{kb}$ (Butler et al. 2009; Pryszcz et al. 2013), whereas the majority of isolates of $C$. orthopsilosis and C. metapsilosis are extremely heterozygous (Pryszcz et al. 2014; Schröder et al. 2016). Most C. orthopsilosis isolates have heterozygosity levels ranging from 8 - 31 SNPs per kilobase, and originated from multiple hybridization (mating) events between related parents (Pryszcz et al. 2014; Schröder et al. 2016). Previous analysis of $11 C$. metapsilosis isolates showed that they were highly heterozygous, ranging from 22 - 26 SNPs per kilobase (Pryszcz et al. 2015). The authors proposed that these $C$. metapsilosis isolates 
arose from hybridization between two parental lineages that differed by approximately $4.5 \%$ divergence at the genome level.

Ten of the 11 C. metapsilosis isolates previously analyzed by (Pryszcz et al. 2015) are heterozygous at the Mating Type-like Locus, with both MTLa and MTLalpha idiomorphs present. The MTLalpha locus is intact, and is identical in the arrangement and orientation of its genes to the MTLalpha locus in C. albicans, C. tropicalis and C. orthopsilosis (Pryszcz et al. 2015). However, introgression has occurred at the MTLa locus, where the PAPa, OBPa and PIKa genes present in most Candida species have been overwritten with MTLalpha2, OBPalpha, and a portion of PIKalpha. Because the introgression is present in almost all sequenced C. metapsilosis isolates, it is likely that hybridization occurred once, followed by introgression, and all extant isolates descended from this. The 11th C. metapsilosis isolate is missing all of MTLa, which Pryszcz et al. (Pryszcz et al. 2015) proposed resulted from an additional $\mathrm{LOH}$ event that has overwritten the remainder of the cassette.

Many fungal species that infect humans are hybrids, including C. orthopsilosis (Pryszcz et al. 2014; Schröder et al. 2016), Candida inconspicua (Mixão et al. 2019) and C. tropicalis (O’Brien et al. 2021). In some fungal pathogens, such as Cryptococcus neoformans (Li et al. 2012), and the plant pathogen Verticillium longisporum (Inderbitzin et al. 2011),

hybridization is associated with increased virulence, increased antifungal resistance or an expanded host range (reviewed in (Mixão and Gabaldón 2018)). In C. orthopsilosis (Schröder et al. 2016) and Cryptococcus neoformans (Xu et al. 2002; Li et al. 2012), multiple hybridization events have occurred and may be ongoing. Here, we describe the discovery of a novel hybrid of $C$. metapsilosis isolated from human feces. This isolate originated from a hybridization event between one parent that is similar to one of the parents of the previously sequenced isolates, and a second parent that is approximately $4.5 \%$ different. We therefore propose that hybridization is also ongoing in the $C$. metapsilosis lineage.

\section{MATERIALS AND METHODS}

DNA extraction and Illumina sequencing. The isolates used in this study are shown in Table S1. Strains from Memorial Sloan Kettering Cancer Center were cultured on Sabouraud (SAB) agar for $48 \mathrm{~h}$ at $37^{\circ} \mathrm{C}$, then grown in overnight culture in $2-3 \mathrm{ml}$ of Yeast ExtractPeptone-Dextrose (YPD) broth at $240 \mathrm{rpm}$. Genomic DNA was extracted and DNA libraries 
were sequenced on an Illumina HiSeq platform generating $100 \mathrm{bp}$ paired-end reads, as described in Zhai et al. (Zhai et al. 2020). Some isolates were previously described in Zhai et al. (Zhai et al. 2020). Illumina data from C. metapsilosis strain ATCC 96143 were downloaded from the NCBI Sequence Read Archive (SRA) under the BioProject ID PRJNA432377 (Oh et al. 2019). Illumina reads from (Pryszcz et al. 2015) were downloaded from the SRA under BioProject ID PRJEB1698. The quality of all Illumina data was checked using FastQC (https://www.bioinformatics.babraham.ac.uk/projects/fastqc/). Reads were trimmed with Skewer version 0.2.2, using parameters "-m pe" (paired end mode) "-1 50" (minimum read length allowed after trimming is 50 bases) “-q 15” (trim 3' end until quality of 15 is reached) “-Q 15" (lowest mean quality allowed before trimming) (Jiang et al. 2014). Data were assembled using SPAdes version 3.13.1 with the --careful parameter (Bankevich et al. 2012).

MinION sequencing. C. metapsilosis MSK414 was cultured on YPD agar for $48 \mathrm{~h}$ at $30^{\circ} \mathrm{C}$, then grown overnight in $50 \mathrm{ml} \mathrm{YPD} \mathrm{broth} \mathrm{at} 30^{\circ} \mathrm{C}, 200 \mathrm{rpm}$. DNA was extracted using the QIAGEN Genomic-Tip $(100 / G)$ kit as per kit instructions. DNA quality was checked with NanoDrop and quantified using the Qubit fluorometer. Libraries were prepared for minION sequencing and barcoded with the Rapid Barcoding Kit (SQK-RBK004) from Oxford Nanopore Technologies (ONT). Prepared libraries from three species were pooled and loaded onto an ONT minION flow cell (FLO-MIN106) for sequencing for $50 \mathrm{~h}$. Basecalling for the minION data was performed using the ONT Guppy software, version 3.2.4 $+\mathrm{d} 9 \mathrm{ed} 22 \mathrm{f}$ with the following parameters; “--input_path fast5 --save_path fastq --flowcell FLO-MIN106 --kit SQK-RBK004 --verbose_logs --cpu_threads_per_caller 5 --num_callers 7’. Basecalled data were demultiplexed using qcat version 1.1.0 with the following parameters; “--fastq fastq/all_multiplexed_reads.fastq --barcode_dir demultiplex_qcat --detect-middle --min-readlength 1 --trim --kit RBK004 --epi2me”. After demultiplexing, $2.79 \mathrm{~Gb}$ of data were assigned to C. metapsilosis MSK414. Read quality was checked with NanoPlot version 1.23.1 (De Coster et al. 2018). Potential contaminant reads (i.e. any reads mapping to the other species sequenced in multiplex on the flow cell) were identified by command-line BLASTN from the BLAST+ package version 2.2.31 (Camacho et al. 2009) and reads with $\mathrm{Q}<7$ and length $<1$ kilobase (kb) were removed with NanoFilt version 2.3.0 (De Coster et al. 2018). Postfiltering, $2.76 \mathrm{~Gb}$ of data assigned to C. metapsilosis MSK414 were available for analysis. 
Filtered MinION reads were assembled using Canu version 1.8 using recommended parameters for haplotype separation; -p canu_run2 -d canu_run2 genomeSize $=13511817$ corOutCoverage $=200$ "batOptions $=-\mathrm{dg} 3$-db3 -dr 1 -ca 500 -cp 50" -nanopore-raw all_q711k.fastq (Koren et al. 2017). Illumina reads were used to polish the assembly with Pilon version 1.23 (Walker et al. 2014). Assembly statistics were checked using QUAST version 4.6.1 (Gurevich et al. 2013). The final assembly consisted of 45 contigs, with 18 chromosomal-sized contigs, an N50 of $1.78 \mathrm{Mb}$, and an L50 of 6 (Table S2). Zeros were removed from the beginning of contig names for clarity. Circos version 0.69 (Krzywinski et al. 2009) and Circoletto (Darzentas 2010) were used to visualize alignments.

Each contig in the C. metapsilosis MSK414 assembly was assigned to either the A or C parent based on its percentage identity to the best hit in the assembly of C. metapsilosis ATCC 96143 (Oh et al. 2019). Global percentage identity was measured using MUMmer dnadiff version 1.3 with default options (Kurtz et al. 2004). Pairs of contigs mapping to one contig in the C. metapsilosis ATCC 96143 reference assembly were assigned as alternative haplotypes of the same chromosome.

Variant calling and filtering. Variants were called from the Illumina data. Trimmed reads were aligned to the chimeric reference assembly produced by (Pryszcz et al. 2015). Reads were aligned using bwa mem version 0.7.12-r1039 with default parameters ( $\mathrm{Li} 2013$ ). Duplicated read alignments were removed using PicardTools MarkDuplicates version 1.95. Variants were called using the Genome Analysis Toolkit (GATK) HaplotypeCaller version 3.7 with default parameters (McKenna et al. 2010). Variants were filtered by removing clusters of variants ( 5 or more variants within 20 bases) using the GATK VariantFiltration tool with parameters “--clusterSize 5 --clusterWindowSize 20". Variants were subsequently filtered for genotype quality (GQ) $<20$ and depth of coverage (DP) $<10$ using GATK VariantFiltration with parameters "--genotypeFilterExpression "GQ $<20$ " -genotypeFilterName GQFilter --genotypeFilterExpression "DP < 10" --genotypeFilterName DPFilter".

For SNP trees, variants were called using the GATK HaplotypeCaller version 3.7 (McKenna et al. 2010) with the additional parameter "--emitRefConfidence GVCF" to produce GVCF files. Joint genotyping was performed for GVCF files from 42 C. metapsilosis strains (Table S1) using the GATK GenotypeGVCFs tool with default parameters. SNPs were extracted 
from the multi-sample VCF and filtered as described above. Repeated Random Haplotype Sampling (RRHS) was used to randomly choose an allele at all heterozygous variant sites and generate a FASTA sequence of all SNPs for each sample (Lischer et al. 2014). This process was completed 1000 times to capture the full breadth of allelic variation in the isolates. Phylogenetic trees were constructed with RAxML version 8.2.9 with the GTRGAMMA model for each of the 1000 SNP sets (Stamatakis 2014). The tree with the best maximum likelihood score was selected as the reference tree, and the remaining 999 trees were used as pseudo-bootstrap trees to generate a supertree.

Loss of Heterozygosity. Heterozygous regions were defined as regions containing at least two heterozygous variants within 100 base pairs (bp) of each other (Pryszcz et al. 2015). Other regions were designated as homozygous. LOH in C. metapsilosis MSK414 was further annotated by aligning Illumina reads to the contigs assigned to the A parent from the Canu assembly with BWA-MEM and calling variants with GATK with parameters as described in 'Variant calling and filtering' (McKenna et al. 2010). LOH regions were annotated as originating from the $\mathrm{C}$ parent if they contained at least one homozygous variant, and as originating from the A parent if there were no homozygous variants in the region. $\mathrm{LOH}$ blocks were plotted using the karyoploteR package in R (Gel and Serra 2017). Divergence between the haplotypes of $C$. metapsilosis MSK414 was calculated as the number of heterozygous variant sites divided by the total length of the heterozygous regions of the genome.

Circos plots. To compare the haplotypes of the diploid C. metapsilosis MSK414 assembly, contigs were first assigned to haplotypes A and C using BLASTN. Circoletto (with Circos version 0.69) (Darzentas 2010). was used to align the 9 largest contigs assigned to haplotype A to the 9 largest contigs from haplotype $\mathrm{C}$ to generate a Circos plot (Krzywinski et al. 2009), with options “--e_value 1e-180 --gep 3 --max_ribbons 10000 --hide_orient_lights -z_by alnlen --untangling_off'. To compare the assembly of C. metapsilosis ATCC 96143 to the diploid C. metapsilosis MSK414 assembly, the 8 largest contigs from the C. metapsilosis ATCC 96143 reference were aligned to the 18 largest contigs from C. metapsilosis MSK414 (including haplotypes A and C), with Circoletto. The same procedure was used to compare the 10 largest contigs from the $C$. metapsilosis chimeric reference assembly to the 18 largest contigs from C. metapsilosis MSK414 (including haplotypes A and C). 


\section{RESULTS}

\section{Population study of $C$. metapsilosis}

The genomes of 11 C. metapsilosis isolates were first sequenced in 2015 (Pryszcz et al. 2015). All were hypothesized to originate from mating between two related, but genetically distinct, individuals. The two parents differed from each other by $\sim 4.5 \%$ at the genome level. A haploid chimeric reference assembly that comprised 57 contigs totaling $13.4 \mathrm{Mb}$ was constructed by combining data from two strains (Pryszcz et al. 2015). Subsequently a collapsed haploid assembly was generated from MinION long read data from C. metapsilosis 96413 (Oh et al. 2019). Here, we carried out a population genomics analysis of $42 C$. metapsilosis isolates, including 11 from Pryszcz et al. (Pryszcz et al. 2015), one from Oh et al (Oh et al. 2019) and 30 from Memorial Sloan Kettering Cancer Center (MSK), of which 25 were described previously (Zhai et al. 2020) (Table S1). Most of the MSK strains were collected as part of a study of a cohort of adult patients with culture-proven fungal bloodstream infections following allogeneic hematopoietic stem cell transplant (allo-HCT). Among these, 26 C. metapsilosis isolates were isolated from a single patient (Zhai et al. 2020). Four were isolated from two other patients with different cancers. DNA was sequenced on an Illumina HiSeq to at least $70 \mathrm{X}$ coverage.

The phylogenetic relationship of the $C$. metapsilosis isolates was determined by constructing trees using SNPs identified across all 42 isolates relative to the chimeric reference genome constructed by Pryszcz et al (Pryszcz et al. 2015). For heterozygous variant sites, one allele was chosen at random using Repeated Random Haplotype Sampling (RRHS) (Lischer et al. 2014). At homozygous variant sites, the alternative allele to the reference was chosen by default. All variant sites were concatenated and SNP trees were drawn using RAxML (Stamatakis 2014). All isolates have high levels of heterozygosity, ranging from 1 heterozygous variant (i.e. heterozygous SNP or indel) per 34 bp to 1 per $49 \mathrm{bp}$, with an average of 1 per 41 bp (Figure 1B). C. metapsilosis MSK414 is distantly related to all other C. metapsilosis isolates (Figure 1A). It is also the most heterozygous isolate analyzed, with 398,389 heterozygous variants (1 every 34 bases) (Figure 1B). C. metapsilosis MSK414 also has a high number of homozygous variants compared to the $C$. metapsilosis chimeric reference assembly (Pryszcz et al. 2015). On average, the $C$. metapsilosis isolates have 53,799 homozygous variants, whereas C. metapsilosis MSK414 has 147,375 homozygous variants (Figure 1B). 
To facilitate a comparison among the other $C$. metapsilosis isolates, SNP trees were drawn excluding C. metapsilosis MSK414 (Figure 1C). 25 of the 26 C. metapsilosis MSK strains (designated by four as the first digit) isolated from a single patient cluster together, as described previously (Zhai et al. 2020). The genomes of these isolates are highly similar (Figure 1C) and could not be differentiated by phylogenetic analysis, although there are some differences in homozygosity levels (Figure S1). Four additional C. metapsilosis isolates from MSK (labeled in green on Figure 1C) cluster separately from the other MSK strains. Isolates described by Pryszcz et al. (Pryszcz et al. 2015) fall into approximately four clades (encircled in Figure 1C), as previously described. C. metapsilosis PL429 does not belong to any clade. C. metapsilosis ATCC 96143, a clinical isolate from Livermore, USA, clusters with one of the groups previously identified by Pryszcz et al. (Pryszcz et al. 2015), together with $C$. metapsilosis MCO448 and C. metapsilosis PL448, which are both clinical isolates from Washington, USA.

\section{Identification of a novel $C$. metapsilosis hybrid}

Figure 1A shows that C. metapsilosis MSK414 is very different to the other C. metapsilosis isolates. We therefore attempted to assemble its genome to facilitate comparison. Previous studies have shown that there are many limitations associated with assembly of short read data from heterozygous diploids (Chan et al. 2012; Zheng et al. 2013; Pryszcz and Gabaldón 2016). During assembly of most diploid genomes, the two haplotypes collapse into a single contig, yielding a haploid assembly. However, for highly heterozygous genomes, this is not possible, and the resulting assemblies are highly fragmented (Pevzner et al. 2001; Li et al. 2010; Gnerre et al. 2011). Pryszcz and Gabaldón (Pryszcz and Gabaldón 2016) developed a protocol (Redundans) that produces a haploid reference assembly by collapsing sequence information from both haplotypes. At heterozygous sites, one allele is randomly chosen to generate one representative contig per diploid chromosome. They assembled a chimeric $C$. metapsilosis haploid genome, using data from two isolates, that has 57 contigs (Pryszcz et al. 2015). However, haplotype information has been lost from this assembly.

We used SPAdes (Bankevich et al. 2012), which keeps haplotypes separate, to assemble the genomes of 42 C. metapsilosis isolates (Table S1). Scaffolds fell into two groups, where the depth of coverage of one group was approximately half of the coverage of the second group. 
Scaffolds with half coverage represent heterozygous regions where both haplotypes have been assembled separately. Scaffolds with high depth of coverage represent homozygous regions that have been collapsed into a single scaffold. This is shown for C. metapsilosis MSK414 (Figure 2) using a coverage-versus-length (CVL) plot (Douglass et al. 2019). This assembly pattern suggests that like all other $C$. metapsilosis isolates, C. metapsilosis MSK414 is a hybrid.

To improve the assembly of C. metapsilosis MSK414, we used Oxford Nanopore MinION long read sequencing. The reads were assembled using Canu (Koren et al. 2017), and errors were corrected by incorporating the Illumina data using Pilon (Walker et al. 2014). This generated an assembly of 45 contigs, with 18 larger than $450 \mathrm{~kb}$, totaling $27 \mathrm{Mb}$ (Table S2). The contigs smaller than $450 \mathrm{~kb}$ were derived from the mitochondrial genome, or from within the chromosomal-sized contigs. A telomeric repeat (ACTTTGGACATCCTAACCTCAAT) was identified at both ends of 14 contigs, and at one end of three of the largest contigs in the assembly. Centromeres (Ola et al. 2020) were identified in 16 contigs, which is consistent with hybridization between two parents with eight chromosomes each (Table S3).

To identify the two haplotypes, we compared the contigs to each other (Figure 3). There is a direct relationship between 13 of the 18 largest contigs. Based on similarities (Figure 3A and Table S3) we assume that tig11866 and tig3 should be joined, and they represent the haplotype A equivalent of tig1 from haplotype $C$ (Figure 3). These 13 contigs therefore represent both haplotypes for 6 of the 8 pairs of $C$. metapsilosis chromosomes. However, the remaining two chromosome pairs are not collinear. tig10 from one haplotype (haplotype A) matches parts of both tig11870 and tig11878 from the second haplotype (haplotype C) (Figure 3A). Similarly, tig11881 from one haplotype (haplotype A) matches part of tig11878 and tig11874 from the second haplotype (haplotype C). Based on similarities, we assume that tig1 1870 and tig 11874 (haplotype C) should be joined (Figure 3B). This is consistent with a single translocation event between the two parental haplotypes (Figure 3B). The translocated chromosomes contain the mating-type like loci (MTL).

To assign the contigs to haplotypes, we compared them to a haploid assembly of $C$. metapsilosis ATCC 96143 (Oh et al. 2019). This assembly is more complete (8 scaffolds) than the original chimeric reference assembly generated by Pryszcz et al (Pryszcz et al. 2015), but still represents a collapsed haploid. In most cases, there is a 1:2 relationship 
between the haploid assembly and the C. metapsilosis MSK414 contigs (Table S3, Figure 3). For each of these, one $C$. metapsilosis MSK414 contig is more similar to the reference (94$96 \%$ identity) and one is less similar (92-93\%). These likely represent the haplotypes of the original parents of MSK414. Contigs 3.1 and 5.1 of C. metapsilosis ATCC 96143 match two contigs in one haplotype of $C$. metapsilosis MSK414 because of the reciprocal translocation (Figure 3B).

We assigned the set of contigs that are more similar to C. metapsilosis ATCC 96143 as haplotype A, and the set of contigs that are less similar to C. metapsilosis ATCC 96143 as haplotype C (Table S3).

\section{Analysis of the Mating-type Like Locus}

Pryszcz et al. (Pryszcz et al. 2015) showed that MTLalpha is intact in 11 C. metapsilosis isolates, and is identical in the order and orientation of its genes to the MTLalpha locus in $C$. albicans, C. tropicalis and C. orthopsilosis. The MTLa locus, however, has been partially overwritten with information from the MTLalpha locus (Figure 4). In MTLa, the PAPa and $O B P \mathbf{a}$ genes have been replaced by the MTLalpha2 and OBPalpha genes. A portion of PIKa has been overwritten with a portion of PIKalpha, producing a chimeric PIK gene at the MTLa. We found the same $M T L$ arrangement in 30 additional $C$. metapsilosis isolates $(C$. metapsilosis ATCC 96143 and 29 C. metapsilosis isolates from MSK). However, $C$. metapsilosis MSK414 has a different organization; both MTLa and MTLalpha are intact (Figure 4).

The MTLalpha locus from C. metapsilosis MSK414 is $\sim 99.8 \%$ identical to the MTLalpha locus from C. metapsilosis ATCC 96143. In addition, the copy of orf19.3202 that is adjacent to MTLalpha is $97 \%$ identical to the reference genome, whereas the copy adjacent to MTLa is only $92 \%$ identical. We therefore assume that MTLalpha was contributed by the same parent, or a very similar parent, in all previously described C. metapsilosis isolates and in $C$. metapsilosis MSK414 (parent A). For most C. metapsilosis isolates, a second parent (parent B) donated the MTLa locus, which has subsequently been overwritten. In C. metapsilosis MSK414 however, MTLa was donated by a third parent, parent $\mathrm{C}$. The majority of $C$. metapsilosis isolates are AB hybrids, whereas C. metapsilosis MSK414 is an AC hybrid. 
Loss of heterozygosity. Previous studies observed that $C$. metapsilosis isolates have undergone large-scale LOH events (Pryszcz et al. 2015). In addition, we found that $C$. metapsilosis isolates MSK403, MSK404 and MSK447 have undergone LOH across most of scaffold 4 (Figure S1). All isolates except for C. metapsilosis MSK414 have undergone significant LOH across part of two scaffolds (Figure S1), supporting the hypothesis that they all descended from the same ancestor. Each C. metapsilosis genome has undergone LOH over approximately half its length (Figure 5A).

The novel hybrid MSK414 stands out as having undergone relatively little LOH (38\% of its length; $5.2 \mathrm{Mb}$ ) (Figure 5A). Regions of $\mathrm{LOH}$ were assigned to either the $\mathrm{C}$ parent (at least one homozygous variant in $100 \mathrm{bp}$ ) or to the A parent (no homozygous variants). LOH regions assigned to parent $\mathrm{C}$ totaled $\sim 5.4 \%$ of the total genome length, while $\mathrm{LOH}$ assigned to the A parent totaled $\sim 26 \%$ of the total genome length. There are 350 blocks of LOH with an average length of $251 \mathrm{bp}$ assigned to the $\mathrm{C}$ haplotype, and 13,892 blocks with an average length of 2,080 bp assigned to the A haplotype. The $\mathrm{LOH}$ tracts are randomly dispersed throughout the contigs (Figure 5B).

\section{DISCUSSION}

Previously sequenced $C$. metapsilosis isolates have an intact MTLalpha locus, with introgression at MTLa (Pryszcz et al. 2015). We found this arrangement in 29 additional isolates from the USA (MSK) and one from Italy (C. metapsilosis ATCC 96143). The relative lack of divergence among these isolates (Figure 1B), and the observation that most share LOH tracts, suggest that they are all derived from the same hybrid ancestor. It is likely that a single ancient hybridization event between A and B parents that differ by $4.5 \%$ was followed by introgression at MTLa, and that most C. metapsilosis isolates descended from this single event.

Despite a lack of evidence at the time, Pryszcz et al. (Pryszcz et al. 2015) suggested that additional hybrid lineages of $C$. metapsilosis may be found. Indeed, analysis of other fungal species, including Cryptococcus neoformans and C. orthopsilosis, showed that hybridization in those species is ongoing and has occurred on multiple separate occasions (Xu et al. 2002; Li et al. 2012; Schröder et al. 2016). However, until now, no different hybrids of $C$. metapsilosis have been identified. Our results show that $C$. metapsilosis MSK414 most likely 
shares one parent (A) with other C. metapsilosis isolates, but its second parent (C) is distinctly different. Parent $\mathrm{C}$ has donated an intact MTLa idiomorph. The A and C haplotypes differ by approximately $4.46 \%$, similar to the divergence between the A and B haplotypes in the other C. metapsilosis isolates (4.5\%; (Pryszcz et al. 2015)). This is also similar to the divergence between haplotypes in hybrids of C. orthopsilosis (Schröder et al. 2016) and $C$. tropicalis (O’Brien et al. 2021).

Identification and separation of parental haplotypes in hybrid species is difficult unless at least one of the parents is known. For C. orthopsilosis, the first genome sequence came, fortuitously, from a highly homozygous strain (C. orthopsilosis 90-125) and so provided a pure reference sequence for the A haplotype of this species (Riccombeni et al. 2012).

Subsequent studies revealed that the majority of $C$. orthopsilosis isolates are, in fact, hybrids between one parent that is essentially identical to this homozygous reference strain, and a second parent that is approximately 4.5\% different from it (Pryszcz et al. 2014; Schröder et al. 2016). For C. metapsilosis MSK, we were able to separate the haplotypes using long read sequencing (ONT).

Pure lineages of the A, B and C haplotypes of C. metapsilosis have not yet been identified. Pryszcz et al (Pryszcz et al. 2015) suggested that only hybrid lineages of Candida species are pathogenic, and that homozygous isolates may only be found in non-clinical samples. This proposal is supported by the observation that most clinical isolates of $C$. orthopsilosis are hybrids (Schröder et al. 2016), and that $C$. albicans is an ancient hybrid (Mixão and Gabaldón 2020). However, rare hybrids of $C$. tropicalis are enriched in environmental, and not clinical sites (O'Brien et al. 2021). Although C. metapsilosis has been isolated from several different body sites, including blood, feces, mucosa, nails, skin, and urine (Hensgens et al. 2009), its natural environment is not known. Hybridization may have enabled $C$. metapsilosis to colonies a new niche, namely the human host. However, "AC" hybrids are rare ( 1 of 42 isolates), and the effect on pathogenicity cannot be fully characterised until environmental isolates are identified.

Our results, together with studies in other species such as C. albicans (Mixão and Gabaldón 2020), C. orthopsilosis (Pryszcz et al. 2014; Schröder et al. 2016), C. tropicalis (O'Brien et al. 2021) and Millerozyma sorbitophila (Louis et al. 2012), suggest that hybridization occurs frequently in members of the CUG-Ser1 clade, and is likely to be current and ongoing. 
Hybridization may represent a mode of adaptation to the host, or possibly to other as yet undetermined conditions.

\section{Data availability.}

All strains are available by request. The raw Illumina data for C. metapsilosis MSK606, MSK607, MSK798, MSK801 and MSK414 are available at accession numbers XXXXX (awaiting accession number). The raw MinION data for C. metapsilosis MSK414 is available at accession number SRR15054248, and the genome assembly is available at BioProject PRJNA730502 (Accession number JAHFZM000000000).

\section{Acknowledgements}

This work was supported by the Wellcome Trust (grant number 109167/Z/15/Z) (G.B.), and

Science Foundation Ireland (grant number 19/FFP/6668) (G.B.), Deutsche

Forschungsgemeinschaft (DFG, German Research Foundation) grant RO-5328/2 (T.R.),

National Institutes of Health (NIH) grants R01 AI093808 (T.M.H.), R21 AI156157 (T.M.H.), P30 CA008748 (Cancer Center Core Grant), the Ludwig Center for Cancer Immunotherapy (T.M.H.), and the Susan and Peter Solomon Divisional Genomics Program (T.M.H.). For the purpose of Open Access, the authors have applied a CC BY public copyright license to any Author Accepted Manuscript version arising from this submission

\section{References}

Bankevich, A., S. Nurk, D. Antipov, A. A. Gurevich, M. Dvorkin et al., 2012 SPAdes: a new genome assembly algorithm and its applications to single-cell sequencing. J. Comput. Biol. 19: 455-477.

Bertini, A., F. De Bernardis, L. A. M. Hensgens, S. Sandini, S. Senesi et al., 2013 Comparison of Candida parapsilosis, Candida orthopsilosis, and Candida metapsilosis adhesive properties and pathogenicity. Int J Med Microbiol 303: 98-103.

Bonfietti, L. X., M. D. A. Martins, M. W. Szeszs, S. B. S. Pukiskas, S. U. Purisco et al., 2012 Prevalence, distribution and antifungal susceptibility profiles of Candida parapsilosis, Candida orthopsilosis and Candida metapsilosis bloodstream isolates. J. Med.

Microbiol. 61: 1003-1008. 
Butler, G., M. D. Rasmussen, M. F. Lin, M. A. S. Santos, S. Sakthikumar et al., 2009

Evolution of pathogenicity and sexual reproduction in eight Candida genomes. Nature 459: 657-662.

Camacho, C., G. Coulouris, V. Avagyan, N. Ma, J. Papadopoulos et al., 2009 BLAST+: architecture and applications. BMC Bioinformatics 10: 421.

Cantón, E., J. Pemán, G. Quindós, E. Eraso, I. Miranda-Zapico et al., 2011 Prospective multicenter study of the epidemiology, molecular identification, and antifungal susceptibility of Candida parapsilosis, Candida orthopsilosis, and Candida metapsilosis isolated from patients with candidemia. Antimicrob. Agents Chemother. 55: 5590-5596.

Chan, G. F., H. M. Gan, H. L. Ling, and N. A. A. Rashid, 2012 Genome sequence of Pichia kudriavzevii M12, a potential producer of bioethanol and phytase. Eukaryot. Cell 11: $1300-1301$.

Darzentas, N., 2010 Circoletto: visualizing sequence similarity with Circos. Bioinformatics 26: $2620-2621$.

De Coster, W., S. D’Hert, D. T. Schultz, M. Cruts, and C. Van Broeckhoven, 2018 NanoPack: visualizing and processing long-read sequencing data. Bioinformatics 34: 2666-2669.

Douglass, A. P., C. E. O’Brien, B. Offei, A. Y. Coughlan, R. A. Ortiz-Merino et al., 2019 Coverage-Versus-Length plots, a simple quality control step for de novo yeast genome sequence assemblies. G3: Genes|Genomes|Genetics g3.200745.2018.

Gel, B., and E. Serra, 2017 karyoploteR: an R/Bioconductor package to plot customizable genomes displaying arbitrary data. Bioinformatics 33: 3088-3090.

Ghannoum, M. A., R. J. Jurevic, P. K. Mukherjee, F. Cui, M. Sikaroodi et al., 2010 Characterization of the oral fungal microbiome (mycobiome) in healthy individuals. PLoS Pathog. 6: e1000713. 
Gnerre, S., I. Maccallum, D. Przybylski, F. J. Ribeiro, J. N. Burton et al., 2011 High-quality draft assemblies of mammalian genomes from massively parallel sequence data. Proc. Natl. Acad. Sci. U. S. A. 108: 1513-1518.

Gomez-Lopez, A., A. Alastruey-Izquierdo, D. Rodriguez, B. Almirante, A. Pahissa et al., 2008 Prevalence and susceptibility profile of Candida metapsilosis and Candida orthopsilosis: results from population-based surveillance of candidemia in Spain. Antimicrob. Agents Chemother. 52: 1506-1509.

Gurevich, A., V. Saveliev, N. Vyahhi, and G. Tesler, 2013 QUAST: quality assessment tool for genome assemblies. Bioinformatics 29: 1072-1075.

Hensgens, L. A. M., A. Tavanti, S. Mogavero, E. Ghelardi, and S. Senesi, 2009 AFLP genotyping of Candida metapsilosis clinical isolates: evidence for recombination. Fungal Genet. Biol. 46: 750-758.

Inderbitzin, P., R. Michael Davis, R. M. Bostock, and K. V. Subbarao, 2011 The ascomycete Verticillium longisporum is a hybrid and a plant pathogen with an expanded host range. PLoS ONE 6: e18260.

Jiang, H., R. Lei, S.-W. Ding, and S. Zhu, 2014 Skewer: a fast and accurate adapter trimmer for next-generation sequencing paired-end reads. BMC Bioinformatics 15: 182.

Koren, S., B. P. Walenz, K. Berlin, J. R. Miller, N. H. Bergman et al., 2017 Canu: scalable and accurate long-read assembly via adaptive -mer weighting and repeat separation. Genome Res. 27: 722-736.

Krzywinski, M., J. Schein, I. Birol, J. Connors, R. Gascoyne et al., 2009 Circos: an information aesthetic for comparative genomics. Genome Res. 19: 1639-1645.

Li, W., A. F. Averette, M. Desnos-Ollivier, M. Ni, F. Dromer et al., 2012 Genetic diversity and genomic plasticity of Cryptococcus neoformans AD hybrid strains. G3 2: 83-97.

Lischer, H. E. L., L. Excoffier, and G. Heckel, 2014 Ignoring heterozygous sites biases 
phylogenomic estimates of divergence times: implications for the evolutionary history of microtus voles. Mol. Biol. Evol. 31: 817-831.

Li, R., H. Zhu, J. Ruan, W. Qian, X. Fang et al., 2010 De novo assembly of human genomes with massively parallel short read sequencing. Genome Res. 20: 265-272.

Lockhart, S. R., S. A. Messer, M. A. Pfaller, and D. J. Diekema, 2008a Geographic distribution and antifungal susceptibility of the newly described species Candida orthopsilosis and Candida metapsilosis in comparison to the closely related species Candida parapsilosis. J. Clin. Microbiol. 46: 2659-2664.

Lockhart, S. R., S. A. Messer, M. A. Pfaller, and D. J. Diekema, 2008b Lodderomyces elongisporus masquerading as Candida parapsilosis as a cause of bloodstream infections. J. Clin. Microbiol. 46: 374-376.

Louis, V. L., L. Despons, A. Friedrich, T. Martin, P. Durrens et al., 2012 Pichia sorbitophila, an interspecies yeast hybrid, reveals early steps of genome resolution after polyploidization. G3 2: 299-311.

McKenna, A., M. Hanna, E. Banks, A. Sivachenko, K. Cibulskis et al., 2010 The Genome Analysis Toolkit: a MapReduce framework for analyzing next-generation DNA sequencing data. Genome Res. 20: 1297-1303.

Mixão, V., and T. Gabaldón, 2020 Genomic evidence for a hybrid origin of the yeast opportunistic pathogen Candida albicans. BMC Biol. 18: 48.

Mixão, V., and T. Gabaldón, 2018 Hybridization and emergence of virulence in opportunistic human yeast pathogens. Yeast 35: 5-20.

Mixão, V., A. P. Hansen, E. Saus, T. Boekhout, C. Lass-Florl et al., 2019 Whole-genome sequencing of the opportunistic yeast pathogen Candida inconspicua uncovers Its hybrid origin. Front Genet 10.:

Németh, T., A. Tóth, J. Szenzenstein, P. Horváth, J. D. Nosanchuk et al., 2013 
Characterization of virulence properties in the C. parapsilosis sensu lato species. PLoS ONE 8: e68704.

O’Brien, C. E., J. Oliveira-Pacheco, E. Ó Cinnéide, M. A. B. Haase, C. T. Hittinger et al., 2021 Population genomics of the pathogenic yeast Candida tropicalis identifies hybrid isolates in environmental samples. PLoS Pathog. 17: e1009138.

Oh, S.-H., B. Smith, A. N. Miller, B. Staker, C. Fields et al., 2019 Agglutinin-Like Sequence (ALS) Genes in the Candida parapsilosis species complex: blurring the boundaries between gene families that encode cell-wall proteins. Frontiers in Microbiology 10.:

Ola, M., C. E. O’Brien, A. Y. Coughlan, Q. Ma, P. D. Donovan et al., 2020 Polymorphic centromere locations in the pathogenic yeast Candida parapsilosis. Genome Res. 30: 684-696.

Pevzner, P. A., H. Tang, and M. S. Waterman, 2001 An Eulerian path approach to DNA fragment assembly. Proc. Natl. Acad. Sci. U. S. A. 98: 9748-9753.

Pryszcz, L. P., and T. Gabaldón, 2016 Redundans: an assembly pipeline for highly heterozygous genomes. Nucleic Acids Res. 44: e113.

Pryszcz, L. P., T. Németh, A. Gácser, and T. Gabaldón, 2014 Genome comparison of Candida orthopsilosis clinical strains reveals the existence of hybrids between two distinct subspecies. Genome Biol. Evol. 6: 1069-1078.

Pryszcz, L. P., T. Németh, A. Gácser, and T. Gabaldón, 2013 Unexpected genomic variability in clinical and environmental strains of the pathogenic yeast Candida parapsilosis. Genome Biol. Evol. 5: 2382-2392.

Pryszcz, L. P., T. Németh, E. Saus, E. Ksiezopolska, E. Hegedűsová et al., 2015 The genomic aftermath of hybridization in the opportunistic pathogen Candida metapsilosis. PLoS Genet. 11: e1005626.

Riccombeni, A., G. Vidanes, E. Proux-Wéra, K. H. Wolfe, and G. Butler, 2012 Sequence and 
analysis of the genome of the pathogenic yeast Candida orthopsilosis. PLoS One 7: e35750.

Schröder, M. S., K. Martinez de San Vicente, T. H. R. Prandini, S. Hammel, D. G. Higgins et al., 2016 Multiple origins of the pathogenic yeast Candida orthopsilosis by separate hybridizations between two parental species. PLoS Genet. 12: e1006404.

Silva, A. P., I. M. Miranda, C. Lisboa, C. Pina-Vaz, and A. G. Rodrigues, 2009 Prevalence, distribution, and antifungal susceptibility profiles of Candida parapsilosis, $C$. orthopsilosis, and C. metapsilosis in a tertiary care hospital. J Clin Microbiol 47: 23922397.

Stamatakis, A., 2014 RAxML version 8: a tool for phylogenetic analysis and post-analysis of large phylogenies. Bioinformatics 30: 1312-1313.

Tavanti, A., A. D. Davidson, N. A. R. Gow, M. C. J. Maiden, and F. C. Odds, 2005 Candida orthopsilosis and Candida metapsilosis spp. nov. to replace Candida parapsilosis groups II and III. J. Clin. Microbiol. 43: 284-292.

Tavanti, A., L. A. M. Hensgens, E. Ghelardi, M. Campa, and S. Senesi, 2007 Genotyping of Candida orthopsilosis clinical isolates by Amplification Fragment Length Polymorphism reveals genetic diversity among independent isolates and strain maintenance within patients. J. Clin. Microbiol. 45: 1455-1462.

Walker, B. J., T. Abeel, T. Shea, M. Priest, A. Abouelliel et al., 2014 Pilon: an integrated tool for comprehensive microbial variant detection and genome assembly improvement. PLoS One 9: e112963.

Xu, J., G. Luo, R. J. Vilgalys, M. E. Brandt, and T. G. Mitchell, 2002 Multiple origins of hybrid strains of Cryptococcus neoformans with serotype AD. Microbiology 148: 203212.

Zhai, B., M. Ola, T. Rolling, N. L. Tosini, S. Joshowitz et al., 2020 High-resolution 

made available under aCC-BY 4.0 International license.

mycobiota analysis reveals dynamic intestinal translocation preceding invasive candidiasis. Nature Medicine 26: 59-64.

Zheng, W., L. Huang, J. Huang, X. Wang, X. Chen et al., 2013 High genome heterozygosity and endemic genetic recombination in the wheat stripe rust fungus. Nat. Commun. 4: 2673. 
bioRxiv preprint doi: https://doi.org/10.1101/2021.07.15.452539; this version posted July $17,2021$. The copyright holder for this preprint A (which was not certified by peer review) is the author/funder, who has granted bioRxiv a license to display the preprint in perpetuity. It is made available under aCC-BY 4.0 International license.
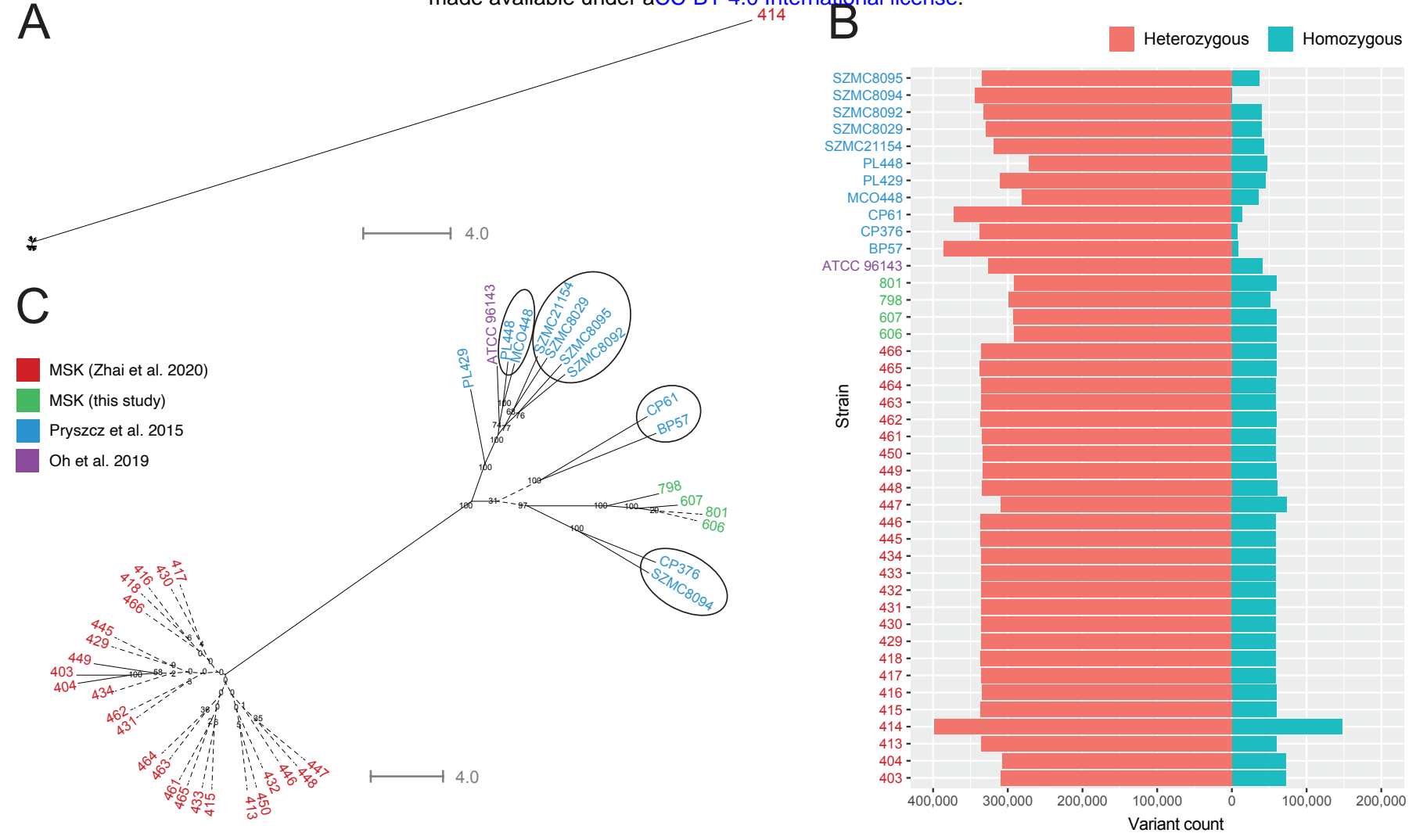

Figure 1. Identification of a divergent C. metapsilosis isolate.

A. C. metapsilosis MSK414 is highly divergent. Phylogenetic SNP trees were generated for 42 clinical C. metapsilosis isolates from various geographical regions (Table S1). SNPs were called using GATK HaplotypeCaller and filtered to remove clusters of variants ( 5 or more variants within 20 bases) and variants with genotype quality $(\mathrm{GQ})<20$ or depth of coverage (DP) $<10$ using the GATK VariantFiltration tool. Repeated Random Haplotype Sampling (RRHS) was used to randomly choose an allele at all heterozygous variant sites and generate a FASTA sequence of all SNPs for each sample (Lischer et al., 2014). In the case of homozygous SNPs, the alternate allele was chosen by default. This process was repeated 1000 times and 1000 phylogenetic trees were constructed with RAxML using the GTRGAMMA model (Stamatakis, 2014). The tree with the best maximum likelihood score was selected as the reference tree, and the remaining 999 trees were used as pseudo-bootstrap trees to generate a supertree. Pseudo-bootstrap values are shown as branch labels. C. metapsilosis MSK414 is labeled in red, while other isolates are not labeled.

B. C. metapsilosis MSK414 has more variants than any other C. metapsilosis isolate. Variant count is shown on the bidirectional $\mathrm{X}$-axis, with heterozygous variants shown on the left in orange and homozygous variants shown on the right in blue. C. metapsilosis strains are labelled on the Y-axis. Isolates from MSK are labelled without the "MSK" prefix. Heterozygosity levels range from 271,440 to 398,389 heterozygous variants. C. metapsilosis MSK414 has more heterozygous variants than all other isolates. Some isolates have almost no homozygous variants, e.g. C. metapsilosis isolates SZMC8094 (used to construct the reference assembly), CP61, CP376 and BP57. C. metapsilosis MSK414 has more heterozygous variants and more than double the number of homozygous variants of any other C. metapsilosis isolate.

C. Other C. metapsilosis isolates fall into two main clusters. Phylogenetic trees for all C. metapsilosis isolates except MSK414 were drawn as in part A. Isolates from MSK are labelled without the "MSK" prefix. Isolates described by Zhai et al. (Zhai et al. 2020) cluster together and are highly similar. Their relationships cannot be accurately resolved (indicated by dashed lines, bootstrap < 40). Four MSK isolates, C. metapsilosis MSK606, C. metapsilosis MSK607, C. metapsilosis MSK798 and C. metapsilosis MSK801, cluster together and are more similar to the clinical isolates described by Pryszcz et al. (Pryszcz et al. 2015) and Oh et al. (Oh et al. 2019) than the other isolates from MSK. The inferred phylogenetic relationships of the isolates analysed by Pryszcz et al. (Pryszcz et al. 2015) fall into four groups, supporting the original analysis, represented by black circles. 
bioRxiv preprint doi: https://doi org/10.1101/2021.07.15.452539. this version posted July 17 2021. The copyright holder for this preprint (which was not certified by peer review) is the author/funder, who has granted bioRxiv a license to display the preprint in perpetuity. It is made available under aCC-BY 4.0 International license.

\section{C. metapsilosis MSK414}

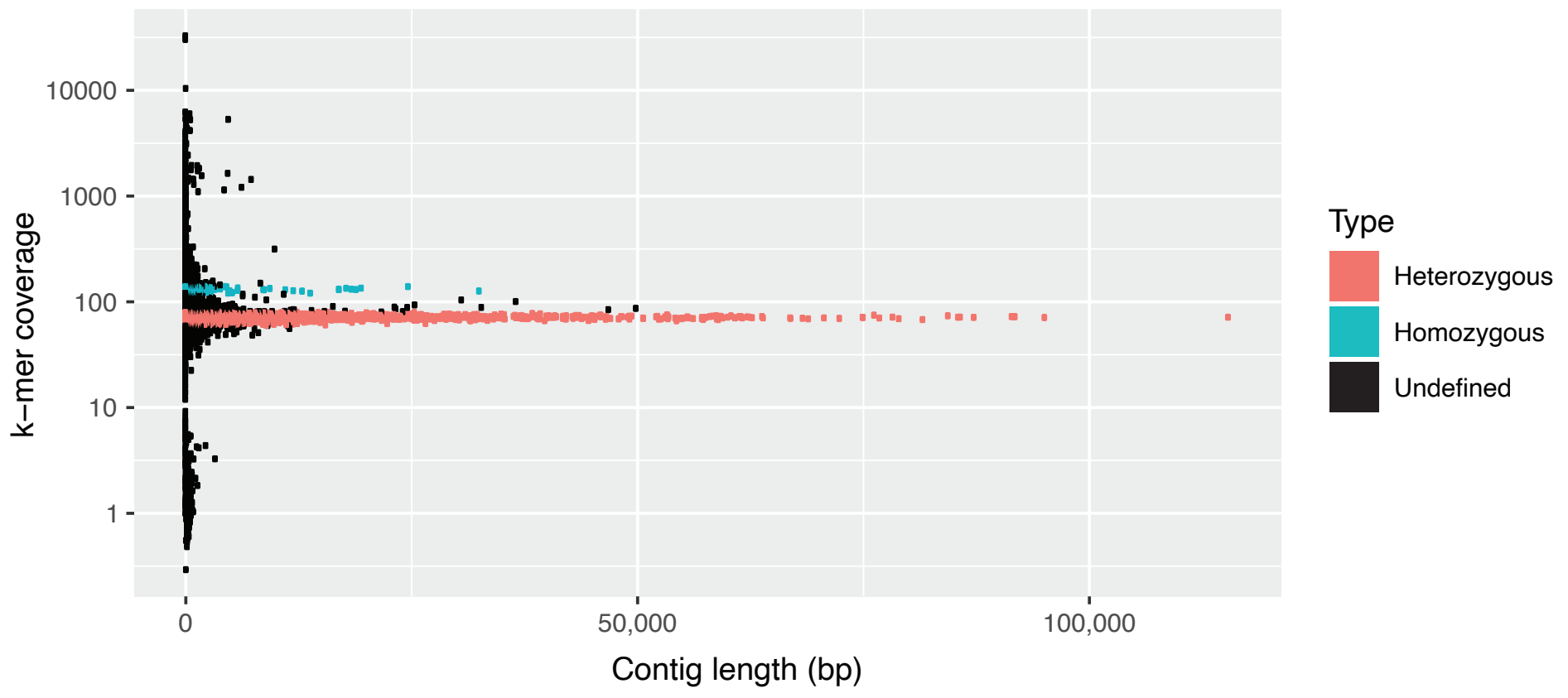

Figure 2. Illumina assembly of C. metapsilosis MSK414 reveals two peaks of coverage.

Scaffolds from the SPAdes assembly of the Illumina data from C. metapsilosis MSK414 are shown as dots. Scaffold length is shown on the X-axis and scaffold k-mer coverage is shown on the Y-axis on a log scale. The majority of the scaffolds have approximately $70 X$ coverage (red). These scaffolds represent heterozygous regions, where both haplotypes have been assembled separately. A second peak of coverage is visible at approximately 130X (cyan). These scaffolds represent homozygous regions that have been collapsed. This structure suggests that C. metapsilosis MSK414 has a hybrid genome (i.e. the two haplotypes are distinctly different). 

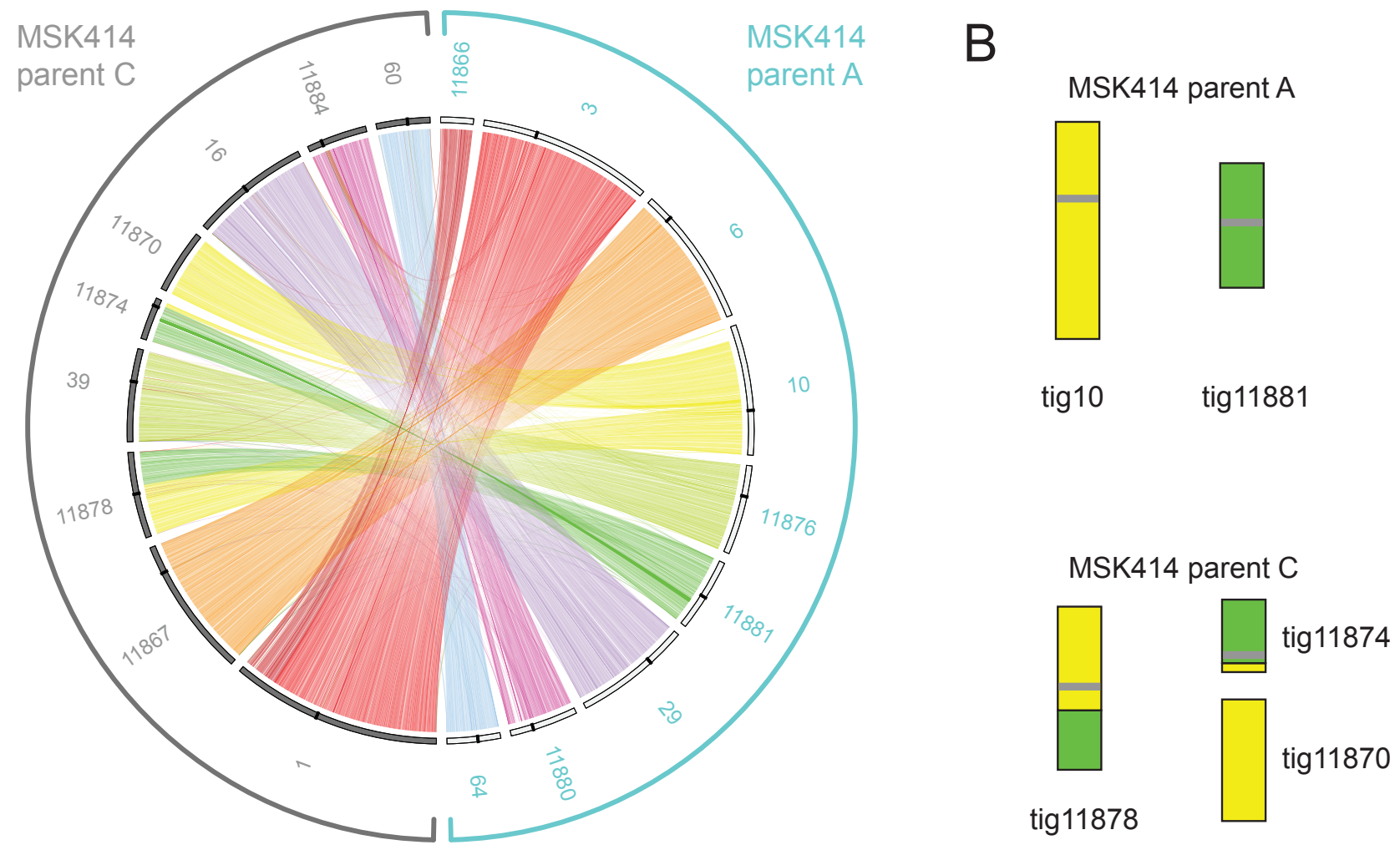

\section{Figure 3. Haplotypes A and C in C. metapsilosis MSK414 differ by one reciprocal translocation.}

A. Similarity between the haplotypes of C. metapsilosis MSK414 was visualized using Circos (Krzywinski et al. 2009) and Circoletto (Darzentas 2010). The 18 largest contigs in the assembly are shown, with the haplotype from the putative A parent on the right (outlined in turquoise) and from the putative C parent on the left (outlined in gray). For clarity, contigs are labelled without the "tig" prefix. Sequences with similarity were identified by BLASTN and alignments with a minimum E-value (1e-180) were plotted as links between the two haplotypes. The 9 largest contigs from parent A (shared with other $C$. metapsilosis isolates) are shown on the right hand side with white bars on the inner layer. The 9 largest contigs from parent $C$ are shown on the left with gray bars on the inner layer. Centromeres are shown as black bars on the inner layer. A translocation is evident between tig 10 and tig11881 in the A haplotype and the equivalent contigs in the $\mathrm{C}$ haplotype.

B. Translocation between tig10 and tig11881 from haplotype $A$ in haplotype $C$ of $C$. metapsilosis MSK414. Contigs in the C. metapsilosis parent A and C haplotypes are shown as colored bars. Centromeres are shown as gray horizontal bars on the contigs. 
MTLa

A. Candida metapsilosis

B. Candida metapsilosis MSK414

C. Candida orthopsilosis
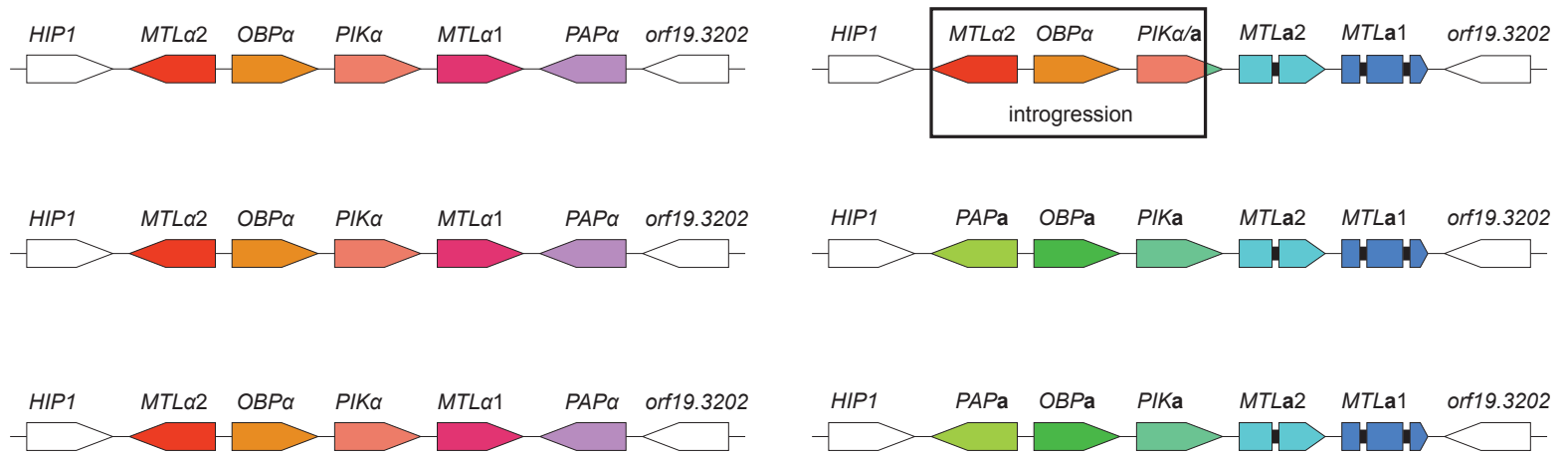

Figure 4. C. metapsilosis MSK414 has a distinct arrangement at MTLa.

The structure of the MTLa and MTLalpha idiomorphs in the majority of $C$. metapsilosis isolates (A), C. metapsilosis MSK414 (B), and C. orthopsilosis (C) are shown, with their relative orientations. Introns in the MTLa1 and MTLa2 genes are shown as black bars. In most C. metapsilosis isolates, the MTLalpha locus is intact, and is identical in its order and orientation to that of $C$. orthopsilosis. The MTLa locus has been partially overwritten by a portion of the MTLalpha locus containing the genes MTLalpha2, OBPalpha, and a part of PIKalpha. The PIK gene is chimeric, comprising part of PIKalpha and part of PIKa. Both MTL idiomorphs in C. metapsilosis isolate MSK414 (B) have the same order and orientation as in C. orthopsilosis (C). In this isolate, the MTLalpha and MTLa idiomorphs are intact with no introgression. 


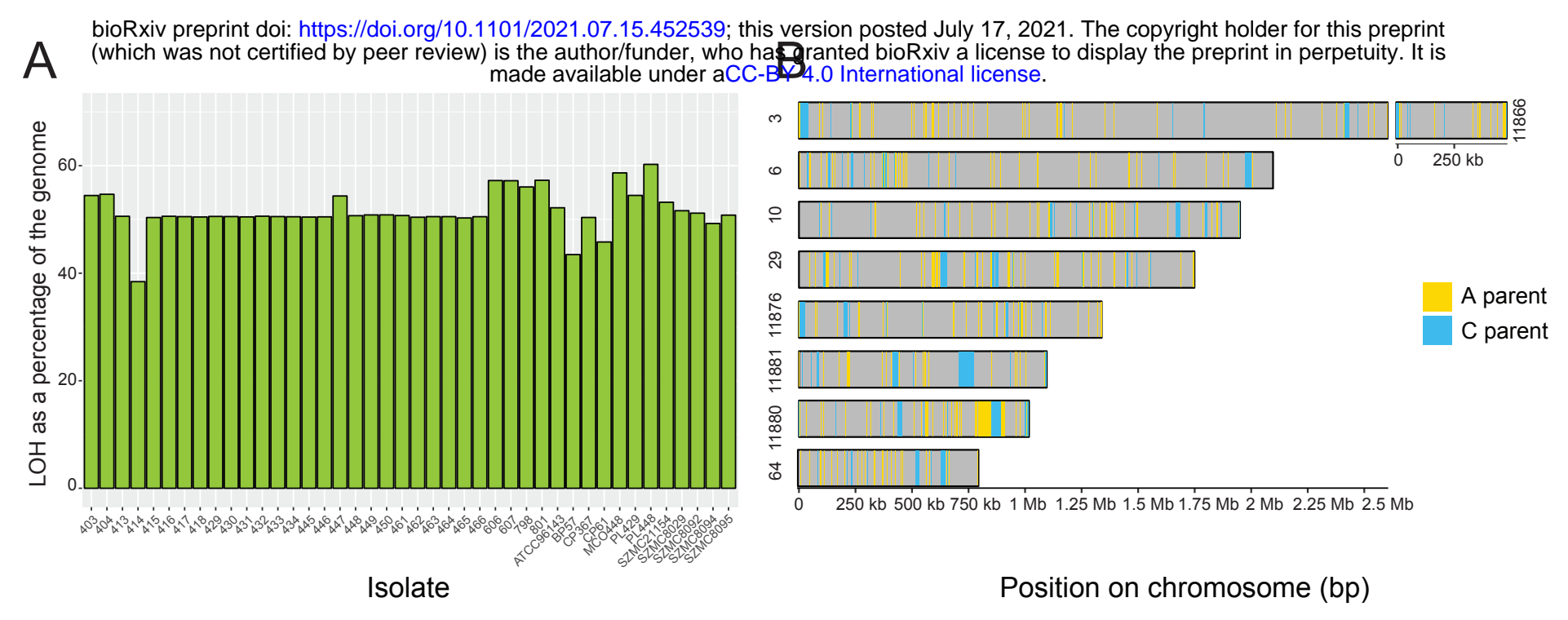

Figure 5. The genome of C. metapsilosis MSK414 has undergone less LOH than other $C$. metapsilosis isolates.

A. Percentage of the genome that has undergone LOH (Y-axis) in C. metapsilosis isolates (X-axis). For most isolates, more than $50 \%$ of the genome has undergone $\mathrm{LOH}$, equating to approximately $6.7 \mathrm{Mb}$. Only 38\% of the C. metapsilosis MSK414 genome has undergone LOH. Two other isolates, C. metapsilosis BP57 and C. metapsilosis CP61, which are closely related (Fig. 1 ), have also undergone less LOH than the other isolates (approximately 43 and $45 \%$ respectively).

B. Regions of LOH in C. metapsilosis MSK414 are dispersed throughout the genome. The nine largest contigs assigned to the C. metapsilosis MSK414 A haplotype are shown. For the sake of clarity, only $\mathrm{LOH}$ regions of at least $1 \mathrm{~kb}$ are illustrated here. Heterozygous regions (defined as any region with at least 2 heterozygous variants within $100 \mathrm{bp}$ of each other), undefined regions, and $\mathrm{LOH}$ regions less than $1 \mathrm{~kb}$ are colored in gray. $\mathrm{LOH}$ blocks were defined as any region of at least 100 bp with fewer than 2 heterozygous variants. LOH regions were assigned to the A parent haplotype (colored in yellow) if there were any homozygous variants present, and to the $\mathrm{C}$ parent haplotype (colored in blue) if there were no homozygous variants. 
bioRxiv preprint doi: https://doi.org/10.1101/2021.07.15.452539; this version posted July 17, 2021. The copyright holder for this preprint (which was not certified by peer review) is the author/funder, who has granted bioRxiv a license to display the preprint in perpetuity. It is $\mathrm{LOH}$ HET made available under aCC-BY 4.0 International license.

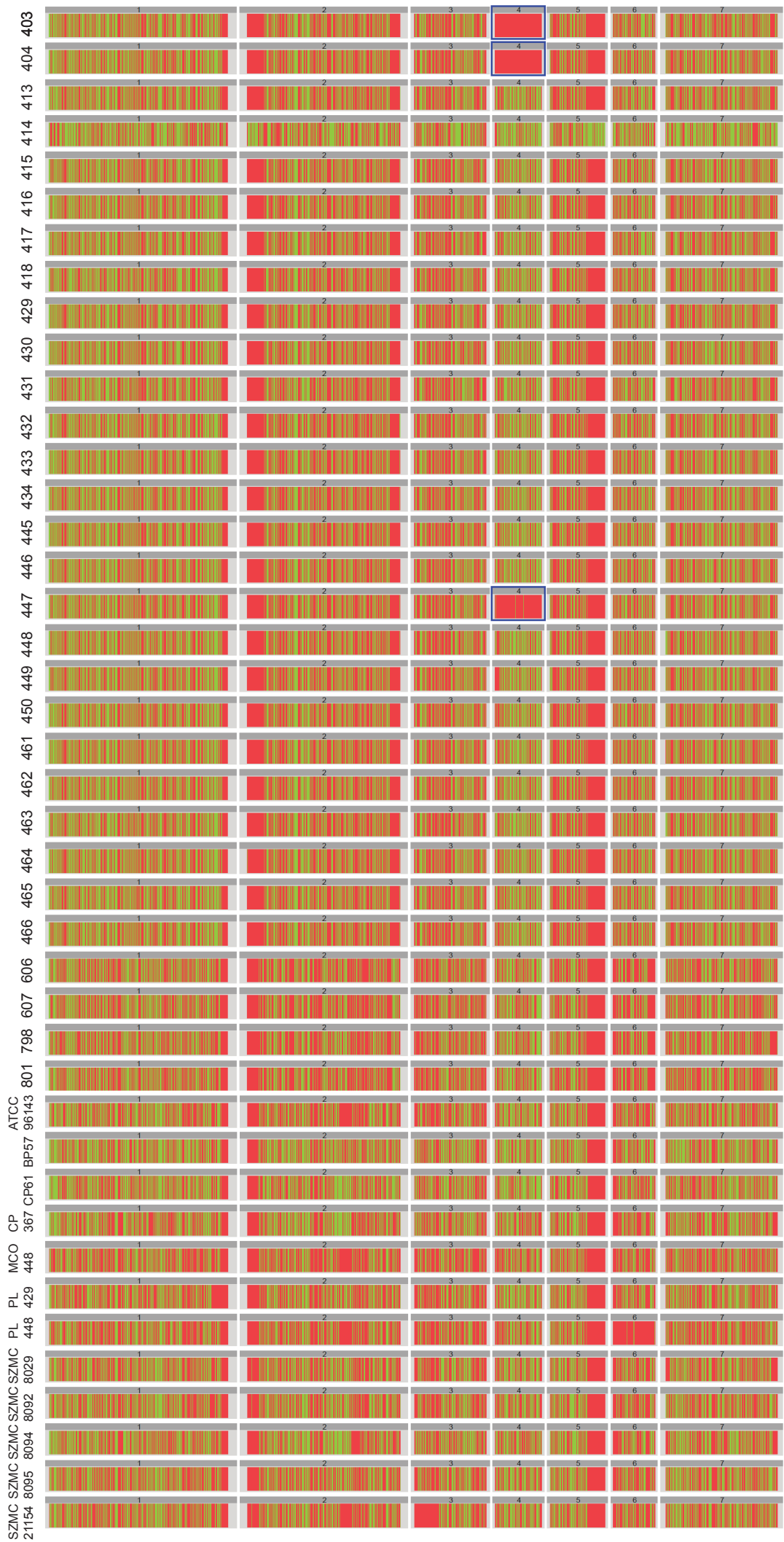

Supplementary Figure 1. Distribution of heterozygous and $\mathrm{LOH}$ regions in the genomes of C. metapsilosis isolates.

The seven largest scaffolds in the chimeric reference genome are displayed horizontally from left to right and labelled from 1 to 7 .

Regions of $\mathrm{LOH}$ are shown in red and heterozygous (HET) regions are shown in green. Isolates are labelled on the left-hand side. MSK isolates are shown without the "MSK" prefix. The genomes of most isolates consist of a mixture of heterozygous and $\mathrm{LOH}$ regions. Isolates 403, 404, and 447 have undergone significant $\mathrm{LOH}$ across most of scaffold 4 (highlighted with blue boxes). Isolate PL448 has undergone significant $\mathrm{LOH}$ on scaffold 6. Large areas of $\mathrm{LOH}$ are visible on scaffolds 2 and 5 in all isolates, except for the new hybrid C. metapsilosis MSK414. 

made available under aCC-BY 4.0 International license.

A

B
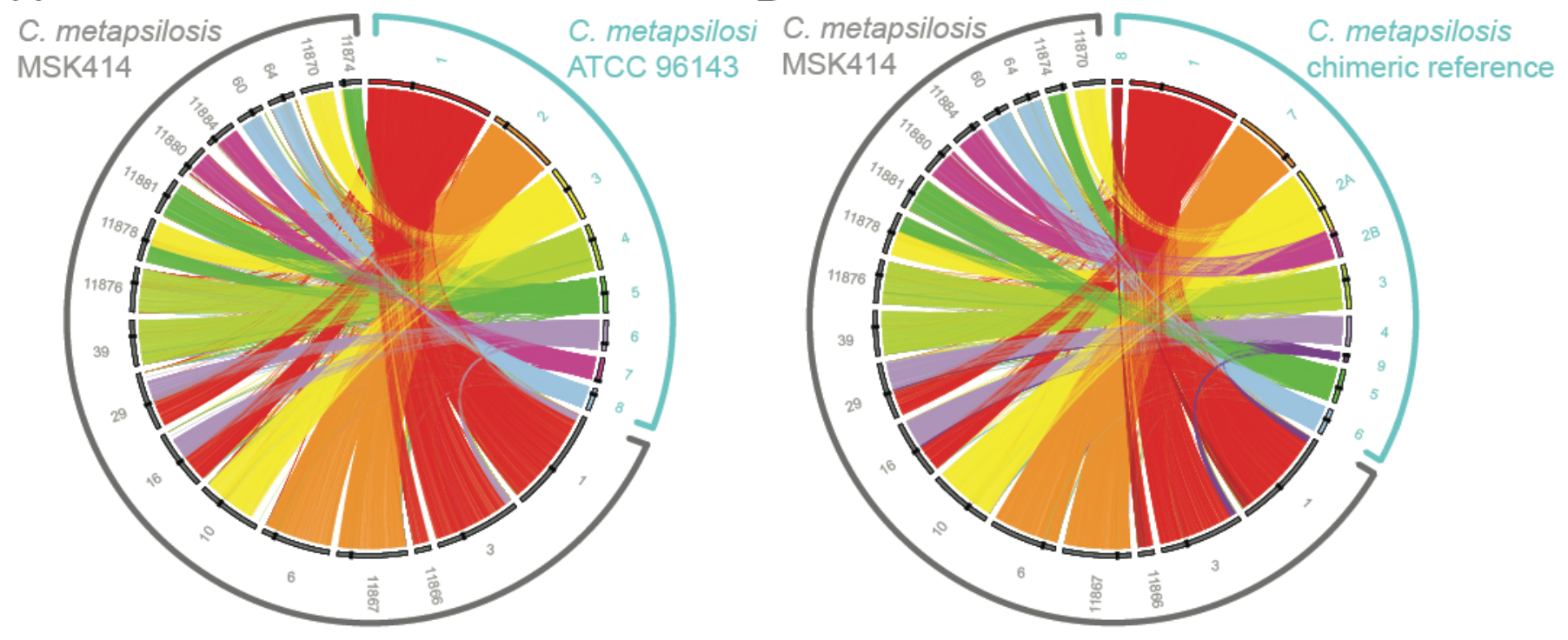

C

PQNC01000001.1

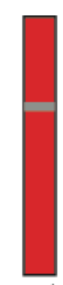

C. metapsilosis ATCC 96143 scaffold1

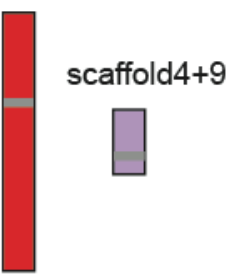

C. metapsilosis chimeric reference

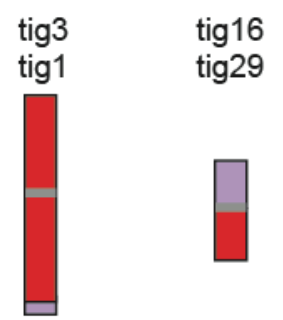

C. metapsilosis MSK414

Supplementary Figure 2. Rearrangements between the diploid assembly of $C$. metapsilosis MSK414 and two haploid C. metapsilosis assemblies.

Sequence similarity between the genome assembly of $C$. metapsilosis MSK414 and the assembly of $C$. metapsilosis ATCC 96143 (A) and the chimeric reference assembly of C. metapsilosis (B) was visualized using Circos (Krzywinski et al. 2009) and Circoletto (Darzentas 2010). Sequences with similarity were identified by BLASTN and alignments with the minimum E- value $(1 e-180)$ were plotted as links between the two assemblies. The 18 largest contigs from the diploid C. metapsilosis MSK414 assembly are represented by grey bars and the "tig" prefix removed for clarity in panels A and B. Centromeres are marked as black bars on the inner layer.

A. Rearrangements between the assembly of C. metapsilosis MSK414 and the assembly of C. metapsilosis ATCC 96143. The eight largest contigs from the haploid C. metapsilosis ATCC 96143 assembly are shown on the upper right hand side of the Circos plot in panel A with colored bars and outlined in turquoise. A mismatch between PQNC01000001.1 and PQNC01000006.1 is observed in both haplotypes of C. metapsilosis MSK414 (tig1 and tig3, and tig16 and tig29). A translocation in one haplotype of C. metapsilosis MSK414 is apparent between PQNC01000003.1 and PQNC01000005.1 (tig11878 and tig11870/tig11874).

B. Rearrangements between the assembly of $C$. metapsilosis MSK414 and the C. metapsilosis chimeric reference assembly. The nine largest contigs from the haploid C. metapsilosis ATCC 96143 assembly are shown on the upper right hand side of the Circos plot in panel B with colored bars and labelled in turquoise. Scaffold 2 contains two centromeres and appears to be the product of an assembly error (supported by the haploid assembly of $C$. metapsilosis ATCC 96143 and tig10 of the C. metapsilosis MSK414 assembly). We refer these as scaffold 2A and scaffold 2B. A mismatch between scaffold 1 and scaffold 4/9 (which we assume should be joined) is visible in both haplotypes of $C$. metapsilosis MSK414 (tig1 and tig3, tig16 and tig29). Scaffolds 4 and 9 most likely represent one chromosome but the assembly has broken at the centromere. This rearrangement mirrors that between scaffolds 1 and 6 of the C. metapsilosis ATCC 96143 assembly.

C. Error in the assembly of C. metapsilosis ATCC 96143 and the chimeric C. metapsilosis reference assembly. Contigs in the C. metapsilosis ATCC 96143 assembly, the chimeric C. metapsilosis reference assembly and the $C$. metapsilosis MSK414 Canu assembly are shown as colored bars. Centromeres are shown as gray horizontal bars on the contigs. The rearrangement observed between PQNC010000001.1 and PQNC010000006.1 is equivalent to the rearrangement between scaffold 1 and scaffold $4 / 9$ in the $C$. metapsilosis chimeric reference. This is likely to be an assembly error in the two haploid assemblies. 
Table S1. C. metapsilosis strains used in this study

\begin{tabular}{|c|c|c|c|c|c|}
\hline Strain ID & Origin & $\begin{array}{c}\text { Site of } \\
\text { Isolation }\end{array}$ & $\begin{array}{c}\text { Read } \\
\text { lengt } \\
\mathbf{h}\end{array}$ & Cov & $\begin{array}{c}\text { Total } \\
\text { variants }\end{array}$ \\
\hline ATCC $96143^{1}$ & Livermore, USA & Unknown & 150 & 42 & 366,857 \\
\hline${\mathrm{BP} 57^{2}}^{2}$ & Pécs, Hungary & Throat & 96 & 151.31 & 394,081 \\
\hline $\mathrm{CP}^{2} 76^{2}$ & Pisa, Italy & Feces & 96 & 163.96 & 345,030 \\
\hline $\mathrm{CP} 61^{2}$ & Pisa, Italy & Nail & 96 & 151.42 & 386,151 \\
\hline $\mathrm{MCO}_{448^{2}}$ & Washington, USA & Hand & 46 & 175.76 & 317,333 \\
\hline PL429² & Livermore, USA & Unknown & 76 & 284.81 & 354,922 \\
\hline PL448² & Washington, USA & Hand & 46 & 186.37 & 318,964 \\
\hline SZMC211154² & Cataluña, Spain & Blood & 100 & 492.44 & 361,914 \\
\hline SZMC8029² & Debrecen, Hungary & Blood & 100 & 425.57 & 369,230 \\
\hline SZMC8092 $^{2}$ & Pisa, Italy & Lung & 100 & 470.78 & 373,057 \\
\hline SZMC8094² & Pisa, Italy & Feces & 100 & 402.91 & 344,840 \\
\hline SZMC8095² & Pisa, Italy & Nail & 100 & 480.67 & 370,999 \\
\hline $\mathrm{MSK} 403^{3}$ & New York, USA & Blood & 101 & 205.47 & 381,084 \\
\hline MSK404 ${ }^{3}$ & New York, USA & Blood & 101 & 248.1 & 379,288 \\
\hline MSK413 3 & New York, USA & Feces & 101 & 262.04 & 394,729 \\
\hline MSK414 & New York, USA & Feces & 101 & 258.03 & 545,764 \\
\hline MSK415 & New York, USA & Feces & 101 & 226.01 & 396,420 \\
\hline $\mathrm{MSK}_{416^{3}}$ & New York, USA & Feces & 101 & 244.2 & 394,311 \\
\hline MSK417³ & New York, USA & Feces & 101 & 285.59 & 394,741 \\
\hline MSK418 & New York, USA & Feces & 101 & 267.76 & 395,401 \\
\hline MSK429 ${ }^{3}$ & New York, USA & Feces & 101 & 314.98 & 394,129 \\
\hline $\mathrm{MSK} 430^{3}$ & New York, USA & Feces & 101 & 293.11 & 394,695 \\
\hline MSK431 ${ }^{3}$ & New York, USA & Feces & 101 & 213.65 & 395,459 \\
\hline MSK432 ${ }^{3}$ & New York, USA & Feces & 101 & 226.58 & 394,260 \\
\hline MSK433 $^{3}$ & New York, USA & Feces & 101 & 257.45 & 394,628 \\
\hline MSK434 ${ }^{3}$ & New York, USA & Feces & 101 & 277.28 & 395,131 \\
\hline MSK445 & New York, USA & Feces & 101 & 297.49 & 395,536 \\
\hline MSK446 $^{3}$ & New York, USA & Feces & 101 & 314.58 & 395,372 \\
\hline MSK4473 & New York, USA & Feces & 101 & 275.69 & 383,033 \\
\hline MSK448 & New York, USA & Feces & 101 & 256.41 & 395,011 \\
\hline MSK4493 & New York, USA & Feces & 101 & 284 & 393,777 \\
\hline
\end{tabular}




\begin{tabular}{|c|c|c|c|c|c|}
\hline MSK450 3 & New York, USA & Feces & 101 & 234.69 & 392,162 \\
\hline MSK4613 & New York, USA & Feces & 101 & 257.75 & 393,295 \\
\hline MSK462 $^{3}$ & New York, USA & Feces & 101 & 284.83 & 395,788 \\
\hline MSK4633 & New York, USA & Feces & 101 & 302.81 & 394,906 \\
\hline MSK464 $^{3}$ & New York, USA & Feces & 101 & 287.72 & 394,569 \\
\hline MSK465 & New York, USA & Feces & 101 & 252.57 & 397,063 \\
\hline MSK466 3 & New York, USA & Feces & 101 & 304.11 & 395,274 \\
\hline MSK606 $^{4}$ & New York, USA & Blood & 101 & 344.82 & 352,155 \\
\hline MSK607 $^{4}$ & New York, USA & Colon & 101 & 358.94 & 352,121 \\
\hline MSK798 ${ }^{4}$ & New York, USA & Blood & 101 & 394.31 & 351,303 \\
\hline $\mathrm{MSK} 81^{4}$ & New York, USA & Blood & 101 & 413.37 & 351,401 \\
\hline
\end{tabular}

${ }^{1}$ (Oh et al. 2019)

2 (Pryszcz et al. 2015)

${ }^{3}$ (Zhai et al. 2020). MSK403-466 were isolated from a single patient.

4 This study. MSK606, 607, 798 and 801 were isolated from two patients. 
bioRxiv preprint doi: https://doi.org/10.1101/2021.07.15.452539; this version posted July 17, 2021. The copyright holder for this preprint (which was not certified by peer review) is the author/funder, who has granted bioRxiv a license to display the preprint in perpetuity. It is made available under aCC-BY 4.0 International license.

Table S2. Comparison of Illumina and minION assemblies of $C$. metapsilosis MSK414.

\begin{tabular}{|l|l|l|}
\hline & Illumina (SPAdes) & \multicolumn{1}{|c|}{ minION (Canu) } \\
\hline Total number of contigs & 13,527 & 45 \\
\hline Total length & $26,170,059$ & $27,138,054$ \\
\hline Largest contig & 115,451 & $3,141,946$ \\
\hline N50 & 22,429 & $1,780,562$ \\
\hline L50 & 337 & 6 \\
\hline & & \\
\hline
\end{tabular}


Table S3. Assignment of contigs in C. metapsilosis MSK414 to haplotypes.

\begin{tabular}{|c|c|c|c|c|}
\hline $\begin{array}{l}\text { ATCC } \\
96143 \\
\text { contig }\end{array}$ & \multicolumn{2}{|c|}{ MSK414 parent A } & \multicolumn{2}{|c|}{ MSK414 parent C } \\
\hline \multirow{15}{*}{$\begin{array}{c}1.1^{1} \\
3,752,469\end{array}$} & \multicolumn{2}{|c|}{$\operatorname{tig}^{\mathrm{ct}}$} & \multicolumn{2}{|c|}{$\operatorname{tig} 1^{\text {tct }}$} \\
\hline & Contig length & $2,603,961$ & Contig length & $3,149,771$ \\
\hline & $\begin{array}{l}\text { Total length of all } \\
\text { alignments }\end{array}$ & $2,367,064$ & $\begin{array}{l}\text { Total length of all } \\
\text { alignments }\end{array}$ & $2,798,055$ \\
\hline & Average identity & 94.81 & Average identity & 92.43 \\
\hline & Identical bases & $2,244,213$ & Identical bases & $2,586,242$ \\
\hline & \multicolumn{2}{|c|}{ tig11866 } & & \\
\hline & Contig length & 488,734 & & \\
\hline & $\begin{array}{l}\text { Total length of all } \\
\text { alignments }\end{array}$ & 479,695 & & \\
\hline & Average identity & 95.75 & & \\
\hline & Identical bases & 459,308 & & \\
\hline & \multicolumn{2}{|c|}{ tig29 $9^{\text {tct }}$} & \multicolumn{2}{|c|}{ tig16 ${ }^{\text {tct }}$} \\
\hline & Contig length & $1,749,045$ & Contig length & $1,784,933$ \\
\hline & $\begin{array}{l}\text { Total length of all } \\
\text { alignments }\end{array}$ & 797,592 & $\begin{array}{l}\text { Total length of all } \\
\text { alignments }\end{array}$ & 775,958 \\
\hline & Average identity & 94.61 & Average identity & 91.86 \\
\hline & Identical bases & 754,602 & Identical bases & 712,795 \\
\hline \multirow{5}{*}{$\begin{array}{c}2.1 \\
2,105,923\end{array}$} & \multicolumn{2}{|c|}{ tig6 $^{\text {tct }}$} & \multicolumn{2}{|c|}{ tig11867 } \\
\hline & Contig length & $2,096,600$ & Contig length & $2,104,178$ \\
\hline & $\begin{array}{l}\text { Total length of all } \\
\text { alignments }\end{array}$ & $2,010,934$ & $\begin{array}{l}\text { Total length of all } \\
\text { alignments }\end{array}$ & $1,980,847$ \\
\hline & Average identity & 94.64 & Average identity & 92.13 \\
\hline & Identical bases & $1,903,148$ & Identical bases & $1,824,954$ \\
\hline \multirow[t]{7}{*}{$\begin{array}{c}3.1^{1} \\
1,700,800\end{array}$} & \multicolumn{2}{|c|}{ tig10 $10^{\text {tct }}$} & \multicolumn{2}{|c|}{$\begin{array}{l}\text { tig11878 }^{\text {tct }} \\
\text { MTLa }\end{array}$} \\
\hline & Contig length & $1,950,477$ & Contig length & $1,289,153$ \\
\hline & $\begin{array}{l}\text { Total length of all } \\
\text { alignments }\end{array}$ & $1,631,051$ & $\begin{array}{l}\text { Total length of all } \\
\text { alignments }\end{array}$ & 729,610 \\
\hline & Average identity & 94.1 & Average identity & 91.70 \\
\hline & Identical bases & $1,534,819$ & Identical bases & 669,052 \\
\hline & & & tig00011870 & \\
\hline & & & Contig length & 954,831 \\
\hline
\end{tabular}




\begin{tabular}{|c|c|c|c|c|}
\hline & & & $\begin{array}{l}\text { Total length of all } \\
\text { alignments }\end{array}$ & 859,421 \\
\hline & & & Average identity & 93.94 \\
\hline & & & Identical bases & 807,340 \\
\hline \multirow{5}{*}{$\begin{array}{c}4.1 \\
1,367,707\end{array}$} & \multicolumn{2}{|c|}{ tig11876 } & \multicolumn{2}{|c|}{ tig39 ${ }^{\text {tct }}$} \\
\hline & Contig length & $1,338,872$ & Contig length & $1,386,788$ \\
\hline & $\begin{array}{l}\text { Total length of all } \\
\text { alignments }\end{array}$ & $1,287,149$ & $\begin{array}{l}\text { Total length of all } \\
\text { alignments }\end{array}$ & $1,267,915$ \\
\hline & Average identity & 94.59 & Average identity & 92.07 \\
\hline & Identical bases & $1,217,514$ & Identical bases & $1,167,369$ \\
\hline \multirow[t]{10}{*}{$\begin{array}{c}5.1^{1} \\
1,080,224\end{array}$} & \multicolumn{2}{|c|}{$\begin{array}{c}\text { tig11881 } \\
\text { MTLt } \\
\text { rDNA }\end{array}$} & \multicolumn{2}{|c|}{ tig11874 $^{\mathrm{t}}$} \\
\hline & Contig length & $1,095,871$ & Contig length & 635,722 \\
\hline & $\begin{array}{l}\text { Total length of all } \\
\text { alignments }\end{array}$ & $1,013,327$ & $\begin{array}{l}\text { Total length of all } \\
\text { alignments }\end{array}$ & 497,399 \\
\hline & Average identity & 95.41 & Average identity & 92.30 \\
\hline & Identical bases & 966,815 & Identical bases & 459,099 \\
\hline & & & \multicolumn{2}{|c|}{$\begin{array}{c}\text { tig00011878 } \\
\text { MTLa }\end{array}$} \\
\hline & & & Contig length & $1,289,153$ \\
\hline & & & $\begin{array}{l}\text { Total length of all } \\
\text { alignments }\end{array}$ & 498,349 \\
\hline & & & Average identity & 91.60 \\
\hline & & & Identical bases & 456,488 \\
\hline \multirow{10}{*}{$\begin{array}{c}6.1^{1} \\
903,567\end{array}$} & \multicolumn{2}{|c|}{ tig29 ${ }^{\text {tct }}$} & \multicolumn{2}{|c|}{ tig16 } \\
\hline & Contig length & $1,749,045$ & Contig length & $1,784,933$ \\
\hline & $\begin{array}{l}\text { Total length of all } \\
\text { alignments }\end{array}$ & 634,361 & $\begin{array}{l}\text { Total length of all } \\
\text { alignments }\end{array}$ & 634,288 \\
\hline & Average identity & 94.7 & Average identity & 91.71 \\
\hline & Identical bases & 600,740 & Identical bases & 581,706 \\
\hline & \multicolumn{2}{|c|}{$\operatorname{tig}^{\mathrm{ct}}$} & \multicolumn{2}{|c|}{ tig1 $^{\text {tct }}$} \\
\hline & Contig length & $2,603,961$ & Contig length & $3,149,771$ \\
\hline & $\begin{array}{l}\text { Total length of all } \\
\text { alignments }\end{array}$ & 216,182 & $\begin{array}{l}\text { Total length of all } \\
\text { alignments }\end{array}$ & 213,770 \\
\hline & Average identity & 94.46 & Average identity & 92.45 \\
\hline & Identical bases & 204,206 & Identical bases & 197,630 \\
\hline \multirow[t]{5}{*}{$\begin{array}{c}7.1 \\
704,064\end{array}$} & \multicolumn{2}{|c|}{ tig11880 } & \multicolumn{2}{|c|}{$\begin{array}{c}\text { tig11884 } \\
\text { rDNA }\end{array}$} \\
\hline & Contig length & $1,017,382$ & Contig length & 904,741 \\
\hline & $\begin{array}{l}\text { Total length of all } \\
\text { alignments }\end{array}$ & 655,192 & $\begin{array}{l}\text { Total length of all } \\
\text { alignments }\end{array}$ & 653,023 \\
\hline & Average identity & 93.79 & Average identity & 92.93 \\
\hline & Identical bases & 614,505 & Identical bases & 606,854 \\
\hline \multirow{2}{*}{$\begin{array}{c}8.1 \\
677,266\end{array}$} & \multicolumn{2}{|c|}{ tig64 $^{\text {tct }}$} & \multicolumn{2}{|c|}{ tig60 ${ }^{\text {tct }}$} \\
\hline & Contig length & 798,529 & Contig length & 802,845 \\
\hline
\end{tabular}




\begin{tabular}{|c|l|l|l|l|}
\hline \multirow{2}{*}{$\begin{array}{c}\text { Total length of all } \\
\text { alignments }\end{array}$} & 592,388 & $\begin{array}{l}\text { Total length of all } \\
\text { alignments }\end{array}$ & 564,780 \\
\cline { 2 - 5 } & Average identity & 94.52 & Average identity & 91.63 \\
\cline { 2 - 5 } Identical bases & $\mathbf{5 5 9 , 9 2 5}$ & Identical bases & $\mathbf{5 1 7 , 5 0 8}$ \\
\hline \multirow{2}{*}{$\begin{array}{c}\text { All } \\
\text { Intigs }\end{array}$} & $\begin{array}{l}\text { Total alignment length } \\
\text { across all contigs }\end{array}$ & $11,684,935$ & $\begin{array}{l}\text { Total alignment length } \\
\text { across all contigs }\end{array}$ & $11,473,415$ \\
\cline { 2 - 5 } & $\begin{array}{l}\text { Identical bases across } \\
\text { all contigs }\end{array}$ & $11,059,795$ & $\begin{array}{l}\text { Identical bases across } \\
\text { all contigs }\end{array}$ & $10,587,038$ \\
\cline { 2 - 5 } & $\begin{array}{l}\text { Average identity } \\
\text { across all contigs }\end{array}$ & $\mathbf{9 4 . 6 5 \%}$ & $\begin{array}{l}\text { Average identity } \\
\text { across all contigs }\end{array}$ & $\mathbf{9 2 . 2 7 \%}$ \\
\hline
\end{tabular}

${ }^{\mathrm{t}}$ Contains a telomere at one or both ends.

${ }^{\mathrm{c}}$ Contains a centromere.

${ }^{1}$ Where contigs resulted from a translocation between parts of two different contigs in the ATCC 96143 assembly, the sequence of the relevant contig was aligned to both contigs in the ATCC 96143 assembly. The average nucleotide identity for each alignment is shown separately. The average identity across all contigs was calculated as the total number of identical bases in all alignments divided by the total length of all alignments across all contigs. There appears to be an assembly error at the tip of scaffold 6 in the assembly of $C$. metapsilosis ATCC 96143 (Oh et al. 2019) (see Figure S2). 